\title{
A model-based framework for the analysis of miniature post-synaptic currents
}

\author{
Marnie A Phillips ${ }^{1,2}$, Laura D Lewis ${ }^{2,4^{*}}$, Martha Constantine-Paton ${ }^{1,3}$, Emery N Brown ${ }^{2,4,5}$ \\ From Nineteenth Annual Computational Neuroscience Meeting: CNS*2010 \\ San Antonio, TX, USA. 24-30 July 2010
}

Miniature post-synaptic currents (mPSCs) have become a primary measure of synaptic modification during development, plasticity, and disease. 'Minis' represent the response of postsynaptic receptors to the spontaneous fusion of vesicles. They are a useful assay for the number and strength of synaptic connections, as mini event frequency is related to the number of functional release sites, and event amplitude is a measure of synapse strength [1]. Thus, accurate characterization of synapse dynamics relies critically on statistical analyses of event frequency and amplitude.

We develop a new paradigm for mPSC analysis that uses likelihood methods [2] and formal goodness-of-fit assessments [3] to derive accurate statistical descriptions of their frequency and amplitude properties. In particular, we demonstrate that MPSC inter-event intervals and amplitudes within individual cells are well described by exponential and log-normal models. These characterizations allow us to analyze mPSCs at the single-cell level. We employ a parametric bootstrap based on these models to make accurate assessments of uncertainty within and between groups in the setting of small samples. This enables accurate estimation of responses for individual cells or groups, and paired comparisons of beforeand after- manipulations in single cells.

We illustrate this approach in the analysis of excitatory mPSCs from acute slices of sensory midbrain. We show that the method may be broadly applicable to excitatory and inhibitory PSCs in other CNS regions, and is robust to changes in event selection parameters and recording conditions.

Our method preserves information about the variability of events within individual cells and allows the summary of information across cells in order to make between-group comparisons. The use of an accurate model maximizes the efficiency of the resulting statistics, by taking advantage of the high degree of structure in the data. The framework allows accurate inferences to be made from studies of spontaneous activity, and for the first time extends analysis of synaptic function to the single cell level.

\section{Acknowledgements}

We are grateful to N. Kuczewski for allowing us to use the hippocampal mIPSC data, and D. Ba, Z.S. Chen and M. Colonnese for helpful comments. This research was supported by NIH grants DP1OD003646, R01EB006385, and R01-EY006039-27, and an FQRNT fellowship.

\section{Author details}

${ }^{1}$ McGovern Institute for Brain Research, Massachusetts Institute of Technology, Cambridge, MA, 02139, USA. ²Department of Brain and Cognitive Sciences, Massachusetts Institute of Technology, Cambridge, MA, 02139, USA. ${ }^{3}$ Department of Biology, Massachusetts Institute of Technology, Cambridge, MA, 02139, USA. ${ }^{4}$ Neuroscience Statistics Research Laboratory, Massachusetts General Hospital, Boston, MA, 02114, USA. ${ }^{5}$ Division of Health Sciences and Technology, Harvard Medical School/MIT, Cambridge, MA, 02139, USA.

\section{Published: 20 July 2010}

\section{References}

1. Hsia AY, Malenka RC, Nicoll RA: Development of excitatory circuitry in the hippocampus. J Neurophysiol 1998, 79:2013-2024.

2. Rice JA: Mathematical statistics and data analysis. Belmont: Thomson/ Brooks/Cole, 2nd 2007.

3. Brown EN, Barbieri R, Ventura V, Kass RE, Frank LM: The time-rescaling theorem and its application to neural spike train data analysis. Neural Comput 2002, 14:325-346.

\section{doi:10.1186/1471-2202-11-S1-P193}

Cite this article as: Phillips et al: A model-based framework for the analysis of miniature post-synaptic currents. BMC Neuroscience 2010 11(Suppl 1):P193.

* Correspondence: Idlewis@mit.edu

${ }^{2}$ Department of Brain and Cognitive Sciences, Massachusetts Institute of

Technology, Cambridge, MA, 02139, USA 